\title{
Consumo de alcohol en la población juvenil
}

\author{
Sánchez Pardo, L. \\ Consejo Asesor del Observatorio Español sobre Drogas
}

Enviar correspondencia: Lorenzo Sánchez Pardo. Delegación del Gobierno para el Plan Nacional sobre Drogas. C/ Recoletos 22. 28001 Madrid. Teléfono 91 5372735. Correo electrónico: Isanchezpa@nexo.es

\section{Resumen}

La serie de Encuestas sobre Drogas a Población Escolar, que realiza desde 1994 la Delegación del Gobierno para el Plan Nacional sobre Drogas entre estudiantes de Secundaria de 14 a 18 años, permite constatar que en 2000 se ha quebrado la expansión de los consumos de alcohol entre los escolares españoles, que venía produciéndose desde los años ochenta, a la vez que en su conjunto se estabilizan los consumos de psicoestimulantes (cocaína y pastillas) y continúan creciendo los de cánnabis. Entre 19982000 se ha producido un descenso del $12 \%$ de los bebedores habituales y un incremento del $10 \%$ de los escolares abstemios, una evolución que se ha visto favorecida por la estabilización de las edades de inicio al consumo (tras años de continuos descensos) y por la intensa reducción de la continuidad en el uso de bebidas alcohólicas.

La equiparación de hábitos entre los sexos (las chicas registran frecuencias de consumo algo superiores, aunque los chicos consumen mayores cantidades) y la concentración de los consumos en el fin de semana, caracterizan los usos de alcohol entre este colectivo. Destacar que, aunque predominan las motivaciones de carácter lúdico, un porcentaje significativo de escolares manifiesta razones relacionadas con la evasión o la superación de problemas personales para explicar el consumo de alcohol. Por otra parte, un $28 \%$ de los escolares que han consumido bebidas alcohólicas reconoce haber sufrido consecuencias negativas asociadas al consumo, desmintiendo la supuesta inocuidad de los usos recreativos del alcohol.

Finalmente, indicar que a pesar de algunos progresos que se han producido en el campo de las percepciones sociales (crece el rechazo generado por los consumos diarios de alcohol), sigue siendo frecuente la banalización de los consumos alcohol (aunque un $20 \%$ se emborrachó en el último mes, sólo un 7\% percibe que bebe bastante o demasiado).

Palabras clave: Epidemiología, alcohol, prevalencias de uso, continuidad de los consumos, consumos recreativos y riesgo percibido.

\section{Summary}

The series of Inquiries about Drugs aimed to Scholar Population, carried out, since 1994, by the Government Delegation for the National Plan about Drugs among 14-18 year old Secondary students, lets us verify that alcohol consumption among Spanish students, which had been spreading since the 80's, has stopped in 2000. Simultaneously, the consumption of psycho-stimulants (cocaine and pills) has stabilized, and the use of cannabis keeps on growing. From 1998 to 2000 there has been a fall of $12 \%$ in regular drinkers and an increase of $10 \%$ in abstemious scholars. This evolution has been helped by the stabilization of the ages of initiation to consumption (after years of constant decrease) and by the intense reduction in the continuity of use of alcoholic drinks.

No relevant differences among genders (girls show a slightly upper frequency in the consumption although boys consume bigger amounts) and concentration of drinking on weekends characterize the uses of alcohol within this group. We have to remark that, although recreational reasons are predominant, an important percentage of scholars reveal reasons related to evasion or to overcoming personal problems to explain their alcohol consumption. On the other hand, $28 \%$ of scholars who have drunk admit negative consequences associated to consumption, challenging the pretended innocuity of recreational alcohol use.

Finally, we have to point out that, despite some progress produced in the field of social perceptions (reject to daily consumptions of alcohol is growing), trivialization keeps being frequent: although $20 \%$ got drunk during last month, only $7 \%$ perceive they drink more than they should.

Key words: Epidemiology, young people, alcohol, prevalence, continuity of consumption, recreational use, perceived risk, Spain. 


\section{INTRODUCCIÓN}

os cambios registrados en las últimas dos décadas en los patrones de consumo de alcohol entre los jóvenes españoles son el reflejo de las profundas transformaciones que ha sufrido la sociedad española en relación con el complejo de las drogas, e ilustran mejor que ninguna otra sustancia las nuevas funcionalidades de lo que ha dado en denominarse como consumos recreativos de drogas. La relevancia epidemiológica del alcohol radica, no sólo en el hecho de que sea la droga más consumida por los adolescentes y jóvenes, sino en el protagonismo que ha adquirido el consumo de bebidas alcohólicas como articulador del tiempo libre de carácter social de estos colectivos, además de su papel como sustancia de referencia en las relaciones de los jóvenes con las drogas.

La crisis del modelo tradicional de consumo de drogas vigente en España hasta mediados de los setenta, supuso la incorporación a los consumos de alcohol y otras drogas de dos colectivos, como las mujeres y los jóvenes, que los valores culturales dominantes hasta ese momento mantuvieron alejados de los mismos. Comienza de este modo un proceso que, apoyado en la progresiva equiparación de hábitos en los consumos de drogas entre los sexos y en la reducción de las edades de inicio al consumo, converge a finales de los ochenta en la expansión de los consumos de alcohol y la generalización de los mismos entre los sectores juveniles ${ }^{1}$.

Las relaciones que los jóvenes españoles mantienen con el alcohol deben contextualizarse en el marco de sus hábitos de ocupación del tiempo de ocio. La asociación entre el consumo de drogas, en especial del alcohol, y diversión ha ido calando entre los jóvenes, hasta convertir el consumo de estas sustancias en un elemento básico de la cultura juvenil y de sus formas de ocio. El consumo de alcohol ha pasado a ser un componente esencial, articulador y dinamizador del ocio de muchos jóvenes, en particular durante las noches del fin de semana ${ }^{2}$. Baste indicar que entre las actividades de ocio practicadas en el fin de semana por los estudiantes españoles figura en segundo lugar el ir de bares o discotecas (el $74^{\prime} 8 \%$ de los encuestados realiza esta actividad), sólo precedida por el salir con amigos/as ${ }^{3}$.

La importante tolerancia social existente todavía respecto a los consumos de alcohol y la escasa percepción del riesgo asociado a la ingesta de bebidas alcohólicas ha sido uno de los factores que ha contribuido a la generalización de los consumos de alcohol entre los adolescentes y jóvenes, favoreciendo la instauración de una imagen de "normalización" de estas conductas, cuando no a la banalización de los consumos ${ }^{4}$. Para verificar la penetración social de este tipo de prácticas basta comprobar que el $34 \%$ de los escolares declara haber salido por las noches todos los fines de semana en el último año, aumentando la frecuencia de las salidas nocturnas con la edad (salen todas las noches del fin de semana el $48^{\prime} 4 \%$ de los estudiantes de 18 años), unas salidas que se prolongan hasta largas horas de la noche y que se acompañan mayoritariamente del consumo de alcohol en locales públicos o en la calle ${ }^{3}$.

La asociación entre el consumo de alcohol y otras drogas y la diversión es un fenómeno generalizado en Europa, como confirman los resultados de un reciente estudio realizado por IREFREA ${ }^{5}$ entre los jóvenes europeos que participan de la vida nocturna, para quienes el alcohol continua siendo la droga favorita, como acredita el que sea consumida, con frecuencia con un patrón abusivo, por cerca del $90 \%$ de los mismos.

Conviene resaltar que el consumo recreativo de alcohol, como definitorio de las relaciones que los jóvenes españoles mantienen con esta sustancia, es en buena medida engañoso, puesto que una parte relevante de los mismos confirma el haber sufrido problemas personales, familiares o sociales como consecuencia del consumo de alcohol. De igual modo, existen numerosas evidencias que señalan que para muchos jóvenes el consumo de drogas ha acabado convirtiéndose 
en un fin en si mismo, sin que de éste se derive una mayor satisfacción personal con su forma de ocupación del tiempo libre ${ }^{6}$. Todo ello sin olvidar el flujo creciente de demandas de tratamiento por abuso del alcohol que formula un número creciente de jóvenes.

Con la finalidad de contribuir a la actualización de las programas dirigidos a reducir la demanda de alcohol y los daños asociados a su consumo entre los adolescentes y jóvenes, así como a la evaluación de los mismos, a continuación se describen los principales resultados aportados por la Encuesta Escolar sobre Drogas 2000, relativos al consumo de alcohol entre los estudiantes de Secundaria. Los datos obtenidos confirman la existencia de una evolución favorable de los consumos de alcohol entre los jóvenes en los últimos años, fruto sin duda de la intensa actividad desplegada para su prevención por diversas instituciones públicas y privadas. Los datos obtenidos ponen de manifiesto la existencia de nuevos valores sociales, de nuevas formas de relaciones familiares y entre los sexos, sin las cuales no resultaría posible comprender las motivaciones que subyacen tras el consumo de bebidas alcohólicas.

\section{LOS CONSUMOS DE ALCOHOL ENTRE LOS ESCOLARES ESPAÑOLES}

El programa de Encuestas Nacionales sobre Drogas que viene desarrollando desde 1994 la Delegación del Gobierno para el Plan Nacional sobre Drogas contempla, entre otras actuaciones, la realización con carácter bianual de una encuesta a Estudiantes de Secundaria con edades comprendidas entre los 14 y los 18 años. La amplitud de las muestras (en la última edición la muestra efectiva estuvo integrada por 20.450 escolares de 596 centros públicos y privados de Secundaria), junto a su estabilidad metodológica, hace de la Encuesta sobre Drogas a la Población Escolar un instrumento valiosísimo para conocer la situación actual de los consumos de las distintas drogas, los patrones de consumo dominantes, las opiniones y actitudes de los escolares respecto a diferentes aspectos relevantes de los consumos, así como de las tendencias observadas desde 1994 en estos ámbitos.

La relevancia de la información facilitada por las distintas Encuestas Escolares sobre Drogas radica en que la población objeto de estudio se corresponde con las edades en las que suelen iniciarse los consumos de drogas y consolidarse ciertas relaciones con las sustancias, permitiendo de este modo la identificación precoz de los hábitos y patrones de consumo emergentes, así como la elaboración de proyecciones respecto a las tendencias de futuro de los mismos.

\subsection{Prevalencia de los consumos de alco- hol}

El alcohol es la sustancia más consumida entre los estudiantes de Secundaria, seguida a bastante distancia del tabaco y el cannabis, como lo confirma el que un $76 \%$ de los escolares haya ingerido bebidas alcohólicas a lo largo de su vida y el que el 58\% lo haya hecho en los últimos 30 días (Tabla 1). Aunque el consumo de alcohol está bastante generalizado entre los escolares, este se concentra fundamentalmente en el fin de semana, así los escolares consumidores de alcohol en los últimos 30 días se distribuyen en dos grandes grupos: un 42'9\% que restringió el consumo en el mes anterior exclusivamente al fin de semana y un $14^{\prime} 7 \%$ que bebió en días laborables y en el fin de semana.

A diferencia de lo que ocurre con las drogas ilícitas, donde en general los consumos que mantienen los escolares se corresponden con un patrón experimental, se observa una elevada continuidad o fidelización en el hábito de ingerir bebidas alcohólicas (sólo superada por el uso de tabaco), como lo acredita el que el 76 '3\% de los escolares que ha consumido alcohol alguna vez en su vida haya repetido su uso en los últimos 30 días. La continuidad en el consumo de alcohol queda también de manifiesto si se analizan 


\section{Tabla 1. Prevalencias de consumo de bebidas alcohólicas entre estudiantes de Secun- daria (14-18 años) y continuidad de uso (porcentaje). España, 2000.}

\begin{tabular}{lcl}
\hline Indicador de uso & Prevalencia & Continuidad de uso \\
\hline Alguna vez en la vida & $76^{\prime} 1$ & Alguna vez - Últimos 12 meses: 98'9\% \\
Últimos 12 meses & $75^{\prime} 3$ & Últimos 12 meses - Últimos 30 días: 77\% \\
Últimos 30 días & 58 & Alguna vez - Últimos 30 días: 76'2\% \\
\hline
\end{tabular}

Fuente: Encuesta sobre Drogas a Población Escolar, 2000. DGPNSD. Observatorio Español sobre Drogas.

las frecuencias de uso, observándose como ya se ha comentado que para uno de cada cuatro escolares que consumen alcohol (indicador "consumo en últimos 30 días") el consumo ha "traspasado" los límites del fin de semana, para trasladarse también a los días laborables.

Por lo que se refiere a la cantidad de alcohol ingerido por los escolares que declaran haber consumido alcohol en los 30 días previos a la realización de la Encuesta, la misma se sitúa como media en el equivalente a 8,4 c.c. de alcohol puro para un día laborable y en 160,9 c.c. para el fin de semana (viernes y sábado). Estos datos confirman la vigencia de un patrón de consumo de alcohol juvenil caracterizado por la concentración de importantes cantidades de alcohol en el fin de semana, como lo acredita el hecho de que casi la mitad de los escolares consumidores de alcohol en los últimos treinta días (el $26 \%$ de los escolares encuestados) consuman 100 o más c.c. de alcohol puro en el fin de semana.

Un indicador indirecto del abuso de alcohol lo constituye la prevalencia y las frecuencias de los episodios de intoxicaciones etílicas (borracheras). Al respecto, apuntar que un $39,7 \%$ de los encuestados declararon haberse emborrachado alguna vez (Tabla 2), tanto más frecuentemente cuanto mayor es la edad del entrevistado (el 15,9\% en el grupo de 14 años y el $65,5 \%$ en el grupo de 18 años). Por lo que respecta a la frecuencia de las borracheras en los últimos 30 días, un $20,6 \%$ de los estudiantes declaran haberse emborrachado en este período, ascendiendo la media a 1'3 borracheras/mes (1,5 para los chicos y 1,2 para las chicas).

A pesar de que los datos anteriores confirman la existencia de un alto porcentaje de escolares que realizan consumos abusivos de alcohol (cercano al 25\%), sólo un 7,4\% considera que bebe bastante o mucho. La importante tolerancia social existente todavía en nuestro país frente al alcohol y el bajo riesgo que los escolares atribuyen al consumo de esta sustancia explican la banalización de los consumos.

Los escolares realizan preferentemente los consumos de alcohol en locales y espacios públicos: pubs o discotecas $\left(78^{\prime} 2 \%\right)$, en la calle o en parques (36'2\%) y en bares o cafe-

\section{Tabla 2. Frecuencia de los episodios de intoxicaciones etílicas entre los estudiantes de Secundaria. España, 2000.}

\section{Prevalencia de los episodios}

Se han emborrachado alguna vez

Se han emborrachado en últimos 30 días

$\mathrm{N}^{\circ}$ de borracheras en últimos 30 días (media) $39^{\prime} 7 \%$

$206 \%$

$1 ' 3$

FUENTE: Encuesta sobre Drogas a Población Escolar, 2000. DGPNSD. Observatorio Español sobre Drogas. 
terías $(32,8 \%)$. Con respecto a las encuestas precedentes se constata un fuerte incremento del consumo en pubs o discotecas, en detrimento del consumo en bares o cafeterías. Estos cambios en los espacios de consumo se relacionan con el progresivo retraso en la hora de regreso a casa en los fines de semana. En este sentido hay que tener en cuenta que el $56 \%$ de los escolares regresó a casa en su última salida en el fin de semana después de las $022^{\prime} 00$ horas (Tabla 3 ).

Tabla 3. Hora de regreso a casa de los estudiantes de Secundaria en la última salida nocturna en el fin de semana. Distribución por edades (porcentaje). España, 2000.

\section{HORA DE REGRESO A CASA LA ÚLTIMA SALIDA EN FIN DE SEMANA}

\begin{tabular}{lcccccc} 
& \multicolumn{7}{c}{ Edad } \\
\cline { 2 - 7 } & $\mathbf{1 4}$ & $\mathbf{1 5}$ & $\mathbf{1 6}$ & $\mathbf{1 7}$ & $\mathbf{1 8}$ & Total \\
\hline Antes de las 12 & 35,0 & 21,5 & 13,0 & 5,6 & 2,5 & 16,2 \\
Entre las 12 y la 1 & 21,3 & 18,3 & 13,3 & 8,1 & 3,8 & 13,9 \\
Entre la 1 y las 2 & 13,3 & 16,6 & 15,3 & 11,5 & 9,0 & 13,9 \\
Entre las 2 y las 3 & 10,9 & 14,5 & 17,4 & 17,7 & 11,1 & 15,1 \\
Entre las 3 y las 4 & 7,0 & 10,3 & 15,2 & 17,6 & 16,2 & 13,2 \\
Después de las 4 & 7,6 & 11,1 & 17,1 & 27,6 & 38,8 & 18,2 \\
La mañana siguiente & 4,9 & 7,7 & 8,7 & 11,8 & 18,7 & 9,4 \\
\hline
\end{tabular}

FUENTE: Encuesta sobre Drogas a Población Escolar, 2000. DGPNSD. Observatorio Español sobre Drogas.

Por lo que respecta a las preferencias de los escolares por las distintos tipos de bebidas, se constata que los combinados/cubatas son las bebidas consumidas en mayor proporción en los últimos 30 días (el 47,3\% lo consumió), seguidos de la cerveza/sidra $\left(30^{\prime} 5 \%\right)$, los licores de frutas $\left(25^{\prime} 8 \%\right)$ y el vino/champán (24'2\%). No obstante, hay que indicar que las preferencias varían según se trate de días laborables o del fin de semana, de modo que la cerveza que es la bebida predominante en los días laborables, es desplazada a un segundo puesto por los combinados en el fin de semana (Tabla 4).

Tabla 4. Tipo de bebidas alcohólicas consumidas por los estudiantes de Secundaria en los últimos $\mathbf{3 0}$ días, distribución por días laborables y fin de semana. España, 2000.

\begin{tabular}{lccc}
\hline & \multicolumn{3}{c}{ Consumo últimos 30 días } \\
\cline { 2 - 4 } & Total & Días Laborables & Fines de Semana \\
\hline Vino/champán & 24,2 & 5,1 & 22,9 \\
Cerveza/sidra & 30,5 & 10,1 & 28,9 \\
Aperitivos & 9,9 & 1,7 & 9,4 \\
Combinados/cubatas & 47,3 & 4,4 & 47,0 \\
Licores de Frutas solos & 25,8 & 3,8 & 25,0 \\
Licores Fuertes solos & 21,7 & 2,2 & 21,4 \\
FUENTE: Encuesta sobre Drogas a Población Escolar, 2000. DGPNSD. Observatorio Español sobre Drogas.
\end{tabular}




\subsection{Consumo de alcohol por sexo y grupo de edad}

La variable sexo establece diferencias significativas en los hábitos de consumo de drogas de los escolares, de modo que la proporción de consumidores de drogas ilícitas es superior entre los chicos, mientras que por el contrario las prevalencias de uso de las sustancias legales, tabaco, alcohol y tranquilizantes, son superiores entre las chicas. Las prevalencias de uso de alcohol son ligeramente superiores entre las chicas, tanto para los consumos experimentales ("alguna vez en la vida"), como para los indicadores consumo en los últimos 12 meses y en los últimos 30 días (Tabla 5).

Aunque las chicas beben en mayor proporción que sus homólogos varones, los chicos ingieren mayores cantidades de alcohol. El consumo medio de alcohol en el fin de semana (viernes y sábado) es el equivalente a $189^{\prime} 6$ cc. de alcohol puro en los chicos y de 130 cc. en las chicas.

\section{Tabla 5. Prevalencias de consumo de alcohol entre los estudiantes de Secundaria según sexo (porcentaje). España, 2000.}

\begin{tabular}{lccc}
\hline SEXO & ALGUNA VEZ & ULTIMOS 12 MESES & ÚLTIMOS 30 DÍAS \\
\hline TOTAL & $76^{\prime} 0$ & $75^{\prime} 3$ & $58^{\prime} 0$ \\
Hombres & $75^{\prime} 6$ & $74^{\prime} 8$ & $57^{\prime} 8$ \\
Mujeres & $76^{\prime} 3$ & $75^{\prime} 8$ & $58^{\prime} 3$ \\
\hline
\end{tabular}

FUENTE: Encuesta sobre Drogas a Población Escolar, 2000. DGPNSD. Observatorio Español sobre Drogas.

La edad condiciona de forma clara las prevalencias de consumo, incrementándose las mismas a medida que lo hace la edad, aunque el crecimiento no es lineal. Los mayores incrementos, tanto para los consumos alguna vez en la vida como en los últimos 30 días se producen entre los 14 y 15 años y los 17 a los 18 años, edades críticas en la progresión de los consumos. Antes de los 16 años, límite de la edad legal para el consumo de alcohol en establecimientos públicos, la mayoría de los escolares ya ha probado las bebidas alco- hólicas (el 71'7\% de los escolares de 15 años). La influencia de la variable edad es más acusada en las frecuencias de uso más próximas, las prevalencias de uso en los 30 días previos a la realización de la Encuesta oscilarían del 30'6\% registrado en los escolares de 14 años al 80\% en los de 18 años.

\subsection{Edades de inicio al consumo \\ El inicio al consumo de alcohol entre los escolares se produce en edades tempranas}

Tabla 6. Prevalencia de los consumos de alcohol entre los estudiantes de Secundaria según edad (porcentaje). España, 2000.

\begin{tabular}{lccc}
\hline EDAD & ALGUNA VEZ & ULTIMOS 12 MESES & ÚLTIMOS 30 DÍAS \\
\hline 14 años & $50^{\prime} 7$ & $49^{\prime} 8$ & $30^{\prime} 6$ \\
15 años & $71^{\prime} 7$ & $71^{\prime} 1$ & $50^{\prime} 5$ \\
16 años & $82^{\prime} 4$ & $81^{\prime} 8$ & $65^{\prime} 0$ \\
17 años & $88^{\prime} 7$ & $88^{\prime} 0$ & $73^{\prime} 5$ \\
18 años & $92^{\prime} 5$ & $92^{\prime} 0$ & $80^{\prime} 0$ \\
\hline
\end{tabular}

FUENTE: Encuesta sobre Drogas a Población Escolar, 2000. DGPNSD. Observatorio Español sobre Drogas. 
(13’6 años como media), estando tan sólo precedido del consumo experimental de tabaco (13'2 años). No obstante, el inicio al consumo semanal de alcohol se retrasa hasta los 14'8 años (Tabla 7). Aunque el primer con- tacto con el alcohol se produce en edades algo más tempranas entre los hombres (13'4 años frente a los 13,8 de las mujeres), la edad de inicio a los consumos semanales es similar para ambos sexos.

Tabla 7. Edad media de inicio al consumo de alcohol entre los estudiantes de Secundaria, según sexo y edad. España, 2000.

\begin{tabular}{|c|c|c|c|c|c|c|c|c|}
\hline & \multicolumn{4}{|c|}{ SEXO } & \multicolumn{3}{|c|}{ EDAD } & \multirow[b]{2}{*}{18} \\
\hline & Total & Hombre & Mujer & 14 & 15 & 16 & 17 & \\
\hline Edad media de consumo por $p$ & 13,6 & 13,4 & 13,8 & 12,4 & 13,1 & 13,7 & 14,2 & 14,5 \\
\hline Edad media de comienzo de consumo semanal & 14,8 & 14,8 & 14,9 & 13,2 & 13,9 & 14,8 & 15,4 & 15,7 \\
\hline
\end{tabular}

FUENTE: Encuesta sobre Drogas a Población Escolar, 2000. DGPNSD. Observatorio Español sobre Drogas.

\subsection{Motivaciones para el consumo y no consumo de alcohol}

Si bien las motivaciones de carácter lúdico siguen siendo mayoritarias entre los escolares para justificar sus consumos de alcohol, emergen con cierta fuerza motivaciones que se relacionan con la existencia de problemas personales o con ciertas dificultades en las relaciones con los demás. Los motivos para consumir alcohol entre los estudiantes que lo hacen habitualmente son, principalmente, porque les gusta su sabor $(73,0 \%)$ y por diversión y placer (58,5\%). Un 15,0\% declara consumir alcohol para olvidar los problemas personales, un $12,9 \%$ para sentir emociones nuevas y un $11,2 \%$ para superar la timidez y relacionarse mejor (Tabla 8). Existen algunas diferencias en las motivaciones expresadas por los dos sexos, siendo por ejemplo superior la proporción de chicas que afirman beber para olvidar los problemas personales (el $17,5 \%$ frente al $12,5 \%$ de los chicos).

Entre las razones para el no consumo destacan los efectos negativos para la salud $(58,5 \%)$, en especial entre los escolares más jóvenes (el $63 \%$ para los de 14 años) y entre los chicos $\left(62,9 \%\right.$ frente al $54^{\prime} 6 \%$ de las chicas). A continuación figuran la pérdida del control producida por el consumo del alcohol y sus efectos desagradables $(45,5 \%)$, su potencialidad para producir accidentes graves $(33,3 \%)$ y que algunos efectos, tales como resaca, mareos o vómitos, sean molestos (30,9\%).

Tabla 8. Motivaciones expresadas por los estudiantes de Secundaria para consumir y no consumir (entre quienes no lo hacen) bebidas alcohólicas. España, 2000.

\begin{tabular}{ll}
\hline $\begin{array}{l}\text { MOTIVACIONES PARA BEBER } \\
\text { (entre quienes beben) }\end{array}$ & $\begin{array}{l}\text { MOTIVACIONES PARA NO BEBER } \\
\text { (de quienes no beben) }\end{array}$ \\
\hline Les gusta el sabor $(73 \%)$ & $\begin{array}{l}\text { Efectos negativos para la salud }\left(58^{\prime} 5 \%\right) \\
\text { Diversión y placer }\left(58^{\prime} 5 \%\right)\end{array}$ \\
$\begin{array}{l}\text { Pérdida de control y efectos desagradables }\left(45^{\prime} 5 \%\right) \\
\text { Olvidar problemas personales }(15 \%)\end{array}$ & $\begin{array}{l}\text { Riesgo de provocar accidentes }\left(33^{\prime} 3 \%\right) \\
\text { Sentir emociones nuevas }\left(12^{\prime} 9 \%\right)\end{array}$ \\
Superar la timidez, relacionarse $\left(11^{\prime} 2 \%\right)$ &
\end{tabular}

FUENTE: Encuesta sobre Drogas a Población Escolar, 2000. DGPNSD. Observatorio Español sobre Drogas. 


\subsection{Problemas asociados al consumo de alcohol}

A pesar de que las motivaciones prioritarias para el consumo de alcohol continúan siendo fundamentalmente lúdicas, una parte muy importante de los escolares reconoce haber sufrido algún problema grave asociado al mismo. Un $28,5 \%$ de los encuestados declaró haber sufrido algún problema o consecuencia negativa como resultado de consumir bebidas alcohólicas a lo largo de su vida. Los problemas citados más frecuentemente son problemas de salud, en un $14,5 \%$ de los casos, riñas y discusiones $u$ otro conflicto sin agresión $(11,1 \%)$, conflictos o discusiones con los padres o hermanos $(10,2 \%)$, problemas económicos $(9,3 \%)$ y peleas o agresiones físicas $(6,3 \%)$. En general se observa un incremento de estos porcentajes conforme aumenta la edad de los entrevistados. Los chicos declaran más a menudo problemas relacionados con el consumo de alcohol, acentuándose la diferencia en las peleas o agresiones físicas $(9,9 \%$ entre ellos y $2,8 \%$ entre ellas).

Destacar que un 19,5\% de los estudiantes que habían consumido alcohol en los doce meses previos a la Encuesta habían conducido durante ese período un vehículo a motor estando bajo los efectos de esta sustancia, o bien habían sido pasajeros de vehículos conducidos por alguien que estaba bajo dichos efectos.

\subsection{Opiniones y actitudes frente al alcohol}

El riesgo percibido por los escolares asociado al consumo de las distintas drogas, la proporción de estudiantes que piensan que esa conducta puede causar bastantes o muchos problemas, aparece claramente asociado a la frecuencia de uso de las sustancias. De tal modo que el riesgo asociado al consumo habitual de cualquier sustancia (entendiendo como tal el consumo diario de tabaco y de alcohol y con una frecuencia de 1 vez o más a la semana para las restantes drogas) es superior al consumo ocasional (consumo 1 vez al mes o con una frecuencia menor), con la única excepción del alcohol (Figura 1).

El consumo habitual de heroína (88'9\%), cocaína $\left(88^{\prime} 3 \%\right)$ y éxtasis $\left(85^{\prime} 2 \%\right)$ son las conductas que los escolares consideran que pueden provocar mayores problemas, mientras que el consumo ocasional de tranquilizantes $\left(32^{\prime} 8 \%\right)$ y cannabis $\left(38^{\prime} 8 \%\right)$ y el diario de alcohol ("tomar 1 ó 2 cañas/copas al día"), con un $42 \%$ de riesgo percibido, son percibidas como las menos problemáticas. El nivel de riesgo asociado al consumo diario de alcohol se encuentra muy alejado del que se atribuye al consumo diario de un paquete de tabaco (73'3\%). En coherencia con el bajo riesgo asociado al consumo diario de alcohol, esta conducta genera el rechazo tan sólo en el $36{ }^{\prime} 8 \%$ de los escolares.

Las razones por las cuales, a juicio de los escolares, el consumo de alcohol puede suponer problemas son: por los efectos que tiene en la salud (75'2\%), porque su consumo provoca accidentes (70'7\%), porque crea adicción $\left(53^{\prime} 6 \%\right)$ y por los problemas familiares derivados de su consumo (37'1\%). Estas razones son en general coincidentes con las esgrimidas para el resto de las sustancias, si bien en este caso la posibilidad de crear adicción se sitúa detrás de los efectos negativos sobre la salud.

Por otra parte, se constata la existencia entre los escolares de un alto grado de disponibilidad percibida con respecto del alcohol, como lo acredita el que el $84 \%$ de los escolares afirmen que les resultaría fácil o muy fácil conseguir esta sustancia, a pesar la corta edad de una parte muy importante de los encuestados.

\section{TENDENCIAS EN LOS CONSUMOS DE ALCOHOL}

La comparación de los resultados obtenidos en la Encuesta Escolar Sobre Drogas 2000, con los aportados por las Encuestas de 1994, 1996 y 1998 permite establecer las tendencias temporales de los consumos de alcohol y de algunos fenómenos asociados a los mismos, que son descritos a continuación. 
Figura 1. Riesgo percibido (proporción de estudiantes que piensan que esa conducta puede causar bastantes o muchos problemas) y rechazo generado por el consumo habitual $(*)$ de las distintas drogas y disponibilidad percibida de las distintas sustancias entre los estudiantes de Secundaria. España 2000.

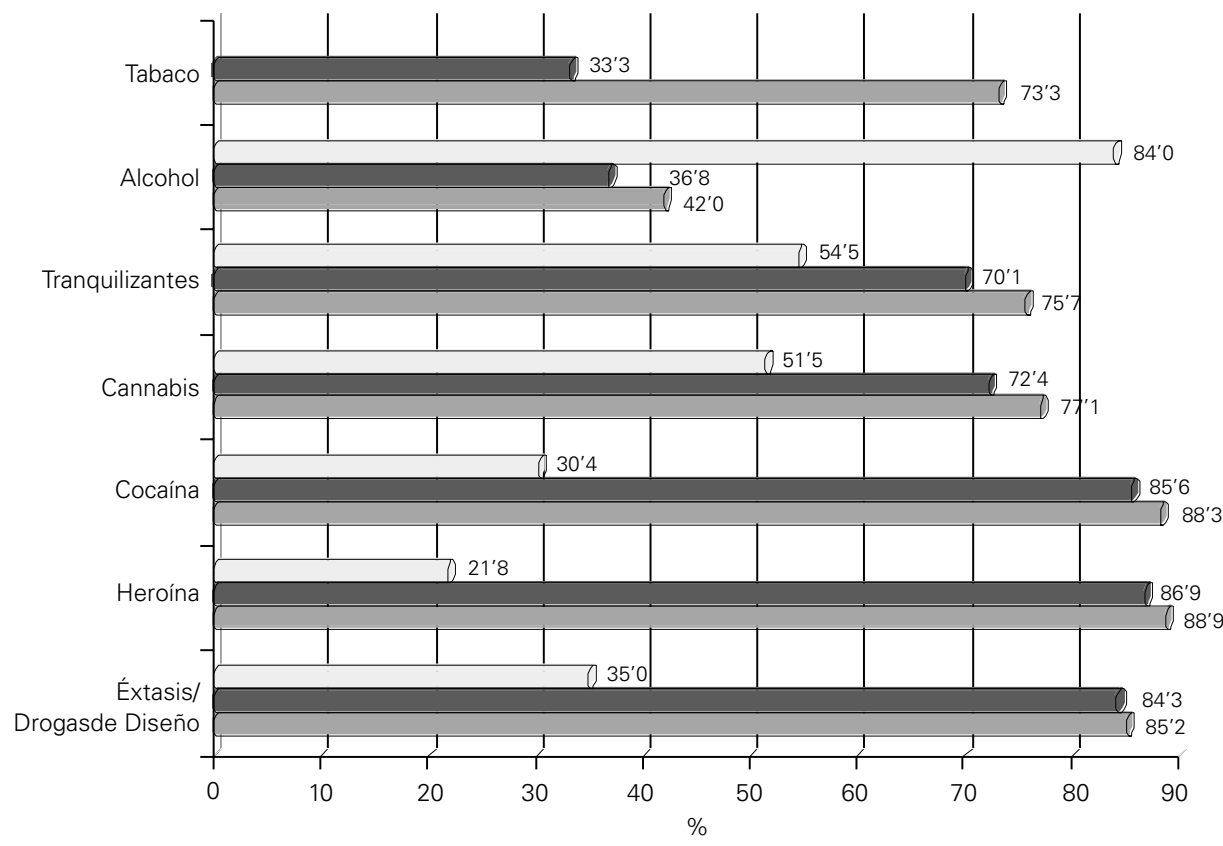

$\square$ Problemas percibidos $\square$ Rechazo $\square$ Disponibilidad

(*) Fumar 1 paquete diario de tabaco, tomar 1 ó 2 cañas-copas al día o consumir 1 vez a la semana o más frecuentemente las distintas drogas.

Fuente: Encuestas sobre Drogas a Población Escolar, 1994, 1996, 1998 y 2000. DGPNSD. Observatorio Español sobre Drogas.

1. El análisis de la evolución de las prevalencias de uso de alcohol en el período 1994-2000 permite constatar la significativa reducción (de 7'7 puntos porcentuales) de los niveles de experimentación con las bebidas alcohólicas, que pasaron del $83^{\prime} 7 \%$ registrado en $1994^{7}$ al $76 \%$ en 2000. Esta reducción ha sido posible gracias al acusado descenso experimentado entre 1998 y 2000 (Figura 2).

2. Mucho más positiva ha sido la evolución registrada por los consumos de alcohol en períodos recientes ("últimos 30 días), que ha visto reducida su prevalencia desde el 78'5\% registrado en 1994 al $58 \%$ del año 2000. Esta favorable evolución de los consumos en los intervalos más recientes, más acusada que los consumos de carácter experimental, ha sido posible gracias a la reducción de la continuidad de uso observada en este período. Si en 1994 un 93'8\% de los escolares que habían ingerido alguna vez bebidas alcohólicas había reiterado el consumo en los últimos 30 días, en el año 2000 la continuidad se redujo al $76^{\prime} 3 \%$. 


\section{Figura 2. Evolución de las prevalencias de uso de alcohol (indicadores consumo alguna vez en la vida, últimos 12 meses y últimos 30 días) entre los estudiantes de Secundaria (porcentaje). España, 1994-2000.}

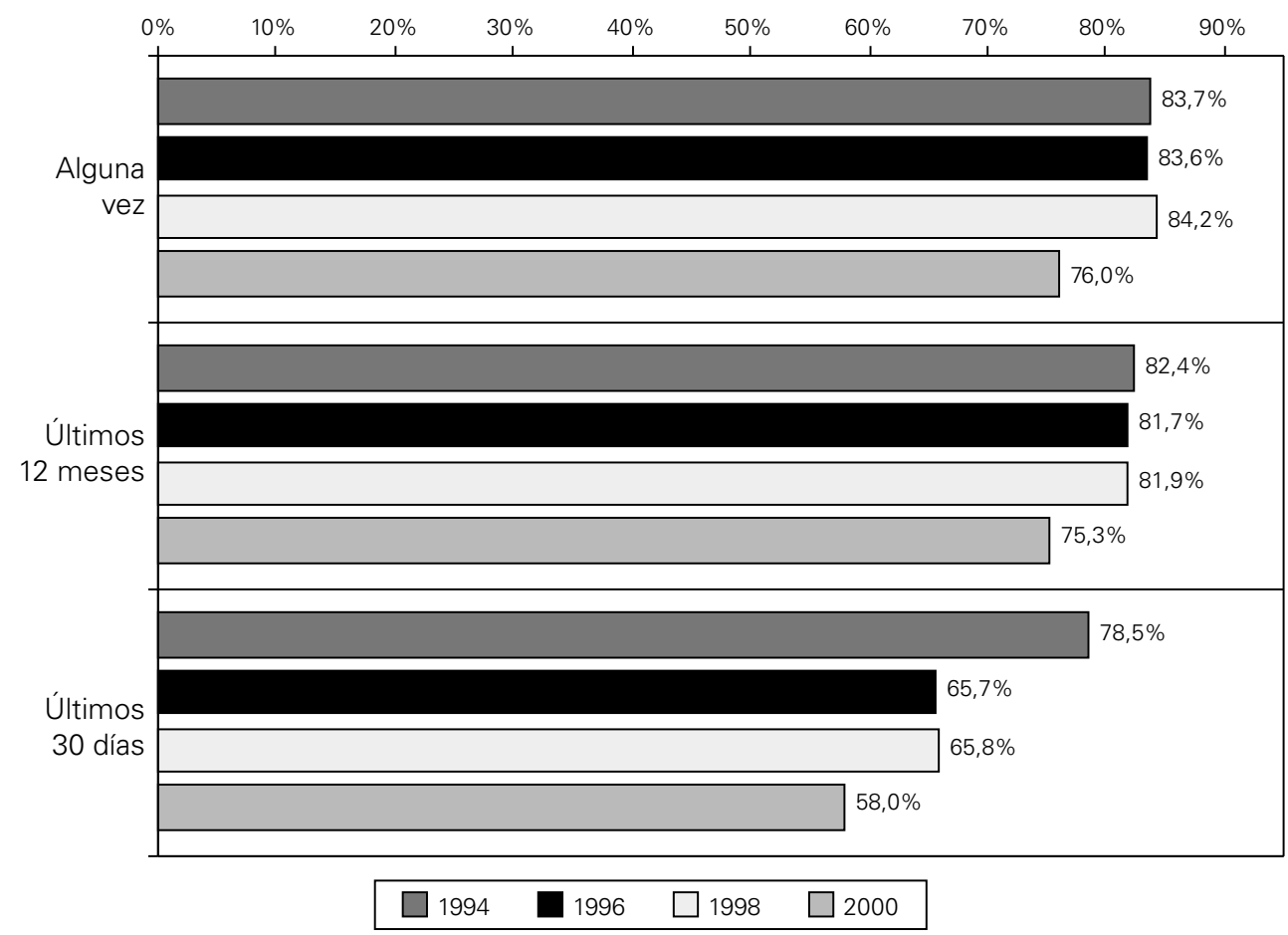

Fuente: Encuestas sobre Drogas a Población Escolar, 1994, 1996, 1998 y 2000. DGPNSD. Observatorio Español sobre Drogas.

3. La evolución de los consumos de alcohol entre 1994-2000 es prácticamente coincidente en ambos sexos, con descensos similares en sus niveles de uso (Figura 3).

4. Por el contrario, se observa una evolución diferencial en los consumos en función de la edad, de modo que los consumos (tanto experimentales, como en los últimos 12 meses y últimos 30 días) se han reducido de forma más intensa entre los escolares más jóvenes (14 y 15 años), mientras que entre los de 18 años únicamente se han reducido para el indicador consumo en los últimos 30 días (Figura 4). El hecho de que la reducción de los consumos de alcohol sea más intensa entre los escolares más jóvenes es un fenómeno altamente positivo y reflejo de los esfuerzos desplegados en el campo de la prevención escolar.

5. Se constata una reducción de los escolares que han sufrido a lo largo de su vida o en los últimos 30 días episodios de intoxicaciones etílicas (borracheras), mientras que crece la proporción de quienes señalan haber sufrido problemas asociados al consumo de alcohol a lo largo de su vida. En el período 1994-2000 no se han producido variaciones en la autopercepción que los escolares tienen de sus propios niveles de ingesta de alcohol, que continúan en niveles bajos (Tabla 9). 
Figura 3. Evolución de las prevalencias de consumo de alcohol entre los estudiantes de Secundaria según sexo (porcentaje). España, 1994-2000.

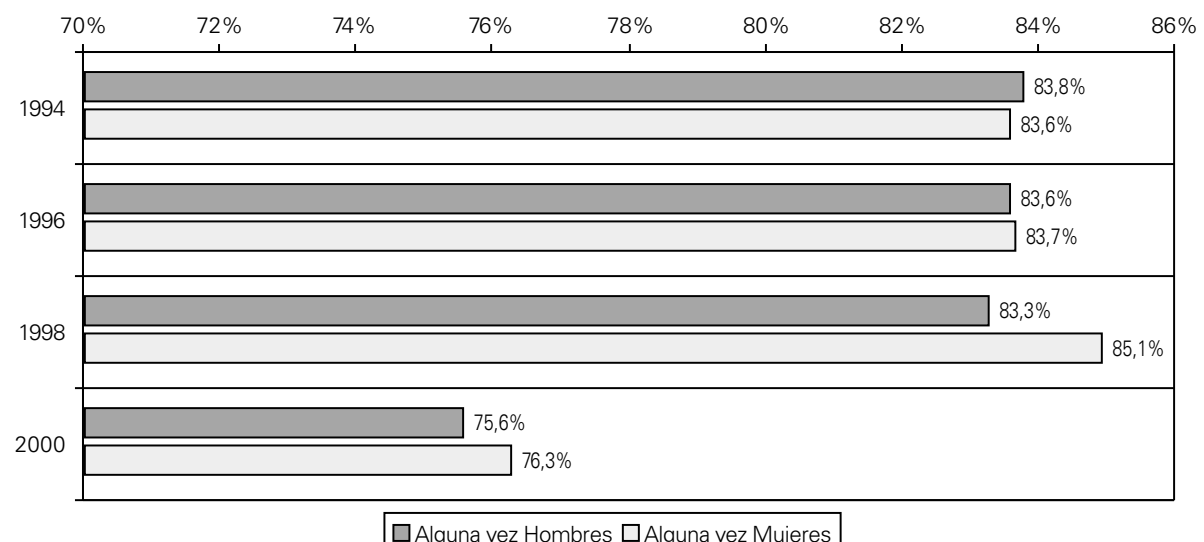

$\square$ Alguna vez Hombres $\square$ Alguna vez Mujeres
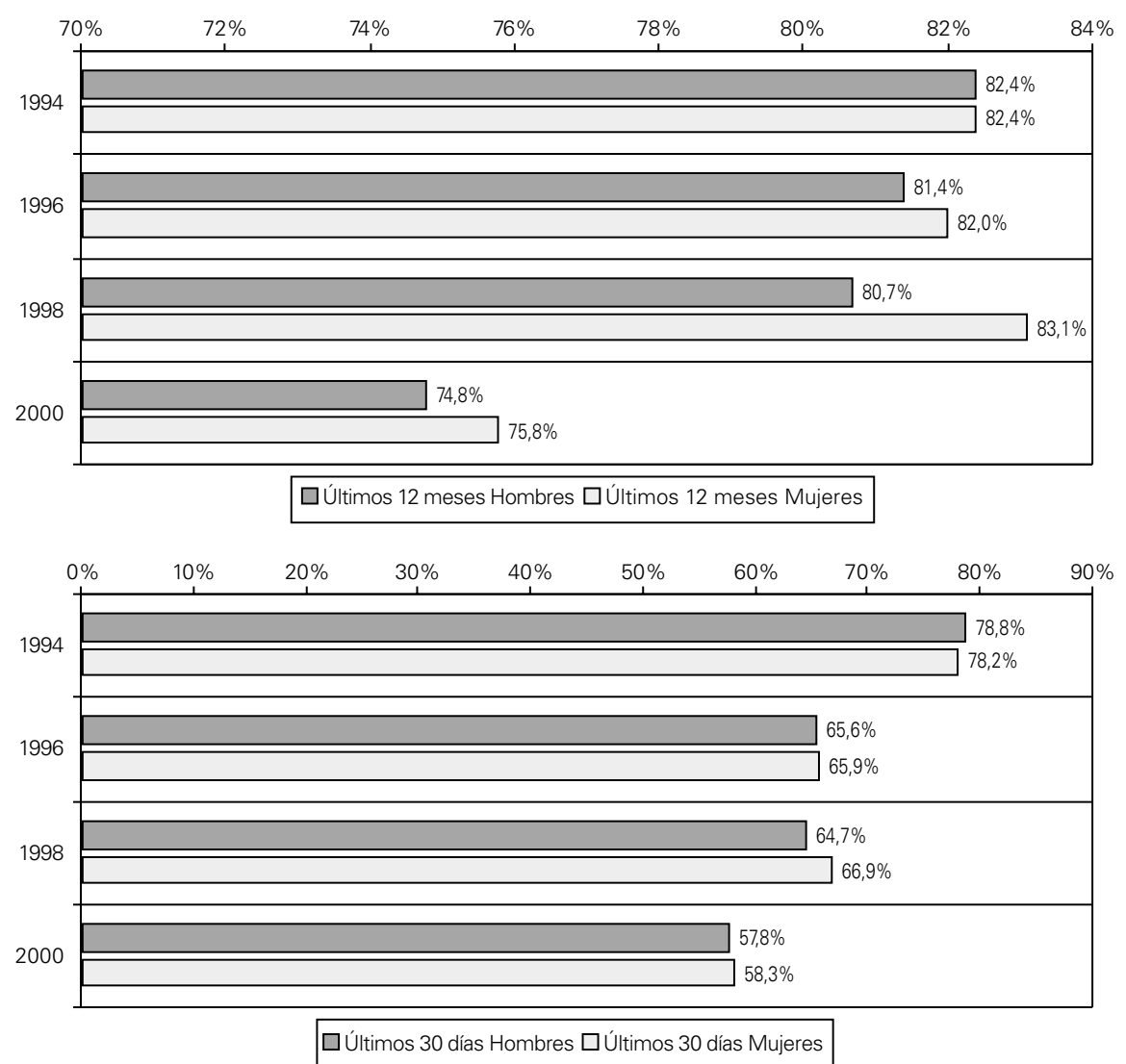

Fuente: Encuestas sobre Drogas a Población Escolar, 1994, 1996, 1998 y 2000. DGPNSD. Observatorio Español sobre Drogas. 
Figura 4. Evolución de las prevalencias de consumo de alcohol entre los estudiantes de Secundaria por edades (porcentaje). España, 1994-2000.
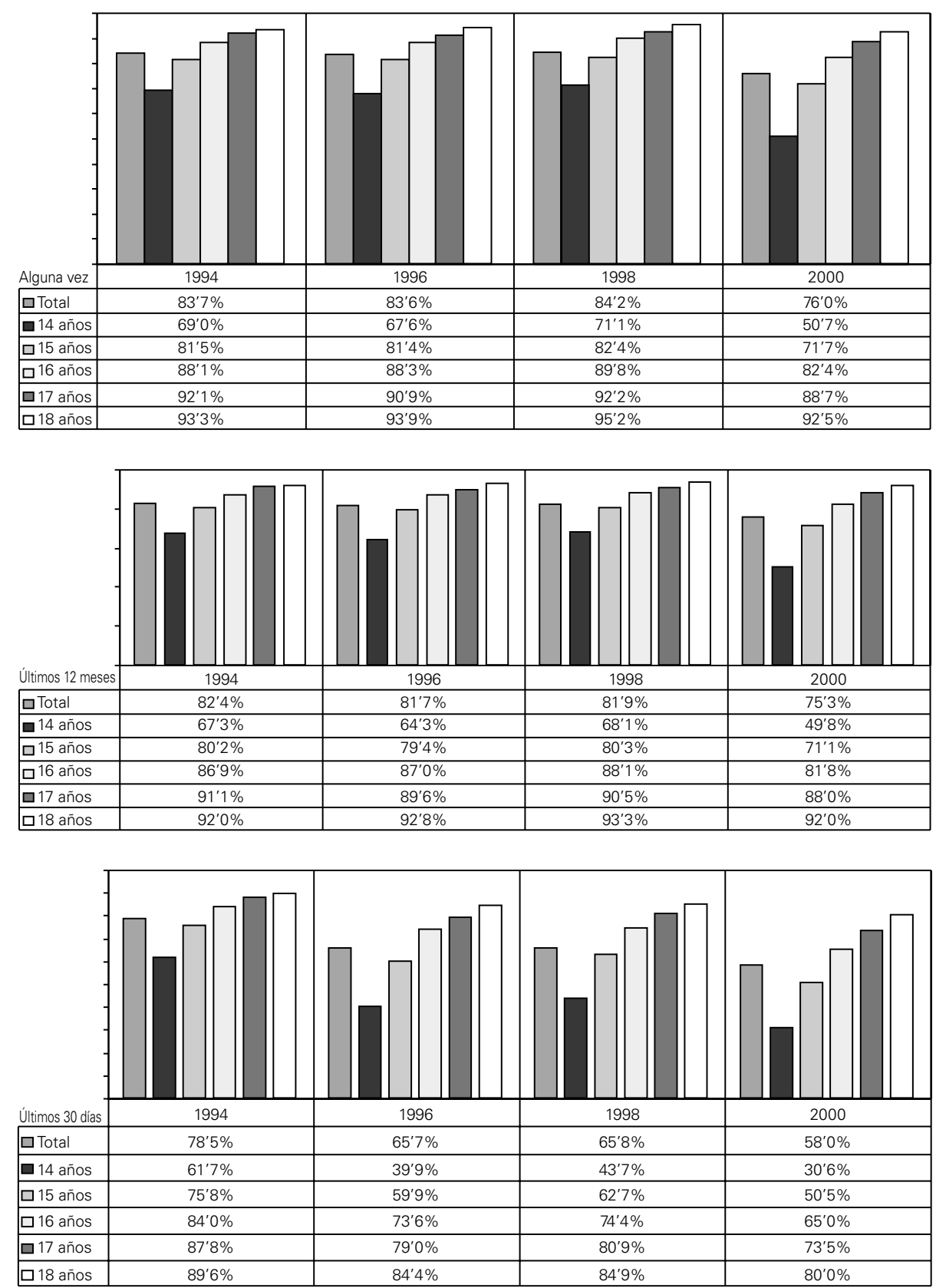

Fuente: Encuestas sobre Drogas a Población Escolar, 1994, 1996, 1998 y 2000. DGPNSD. Observatorio Español sobre Drogas. 
Tabla 9. Evolución de los estudiantes de Secundaria con problemas relacionados con el consumo de alcohol (porcentaje). España, 1994-2000.

\begin{tabular}{lcccc}
\hline & $\mathbf{1 9 9 4}$ & $\mathbf{1 9 9 6}$ & $\mathbf{1 9 9 8}$ & $\mathbf{2 0 0 0}$ \\
\hline Menciona problemas asociados al consumo & & $22^{\prime} 4$ & $24^{\prime} 5$ & $28^{\prime} 5$ \\
Se ha emborrachado alguna vez en la vida & $43^{\prime} 5$ & $40^{\prime} 5$ & $41^{\prime} 0$ & $39^{\prime} 7$ \\
Se ha emborrachado alguna vez últimos 30 días & $22^{\prime} 5$ & $21^{\prime} 4$ & $23^{\prime} 6$ & $20^{\prime} 6$ \\
Perciben que beben bastante o mucho alcohol & $7^{\prime} 2$ & $7^{\prime} 2$ & $8^{\prime} 5$ & $7^{\prime} 4$ \\
\hline
\end{tabular}

FUENTE: Encuestas sobre Drogas a Población Escolar, 1994, 1996, 1998 y 2000. DGPNSD. Observatorio Español sobre Drogas.

6. En el período $1994-2000$ se han retrasado ligeramente las edades medias de inicio al consumo de alcohol (desde los $13^{\prime} 4$ a los 13'6 años), gracias al comportamiento más favorable registrado por los chicos (la edad de inicio al consumo de las chicas se ha mantenido inalterable). Sin embargo, debe destacarse que desde 1996 este indicador se encuentra prácticamente estabilizado (Figura 5).

Figura 5. Evolución de las edades medias de inicio al consumo de alcohol por sexo (primer consumo y consumo con una frecuencia semanal) de los estudiantes de Secundaria (años). España 1994-2000.

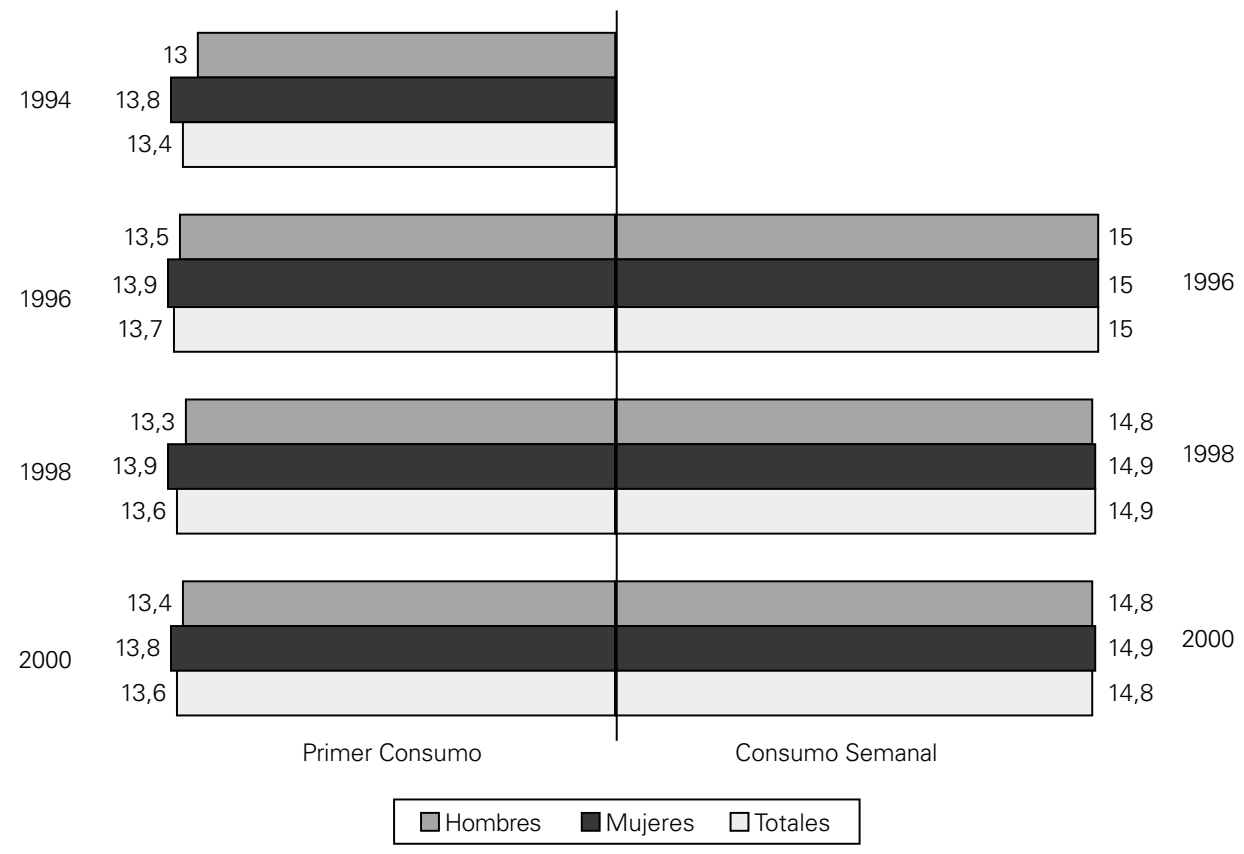

Fuente: Encuestas sobre Drogas a la Población Escolar, 1994, 1996, 1998 y 2000. DGPNSD. Observatorio Español sobre Drogas. 
7. Por lo que respecta a la evolución registrada en las opiniones y actitudes de los escolares frente a diversos aspectos o dimensiones relacionados con los consumos de alcohol, apuntar que han seguido tendencias contradictorias desde 1994 (Figura 6). Mientras el riesgo asociado al consumo habitual de alcohol (tomar 1 ó 2 cañas/copas al día) se ha reducido (del $47^{\prime} 4$ al $\left.42 \%\right)$, ha crecido ligeramente el rechazo que genera esa misma conducta (del 35'5 al 36'8\%) y se ha reducido la disponibilidad percibida del alcohol (del 88 al $84 \%)$.

\section{Figura 6. Evolución del riesgo percibido (proporción de estudiantes que piensan que esa conducta puede causar bastantes o muchos problemas) y del rechazo generado por el consumo diario de alcohol ( 1 ó 2 cañas/copas al día) y la disponibilidad percibida de alcohol entre los estudiantes de Secundaria. España 1994-2000).}

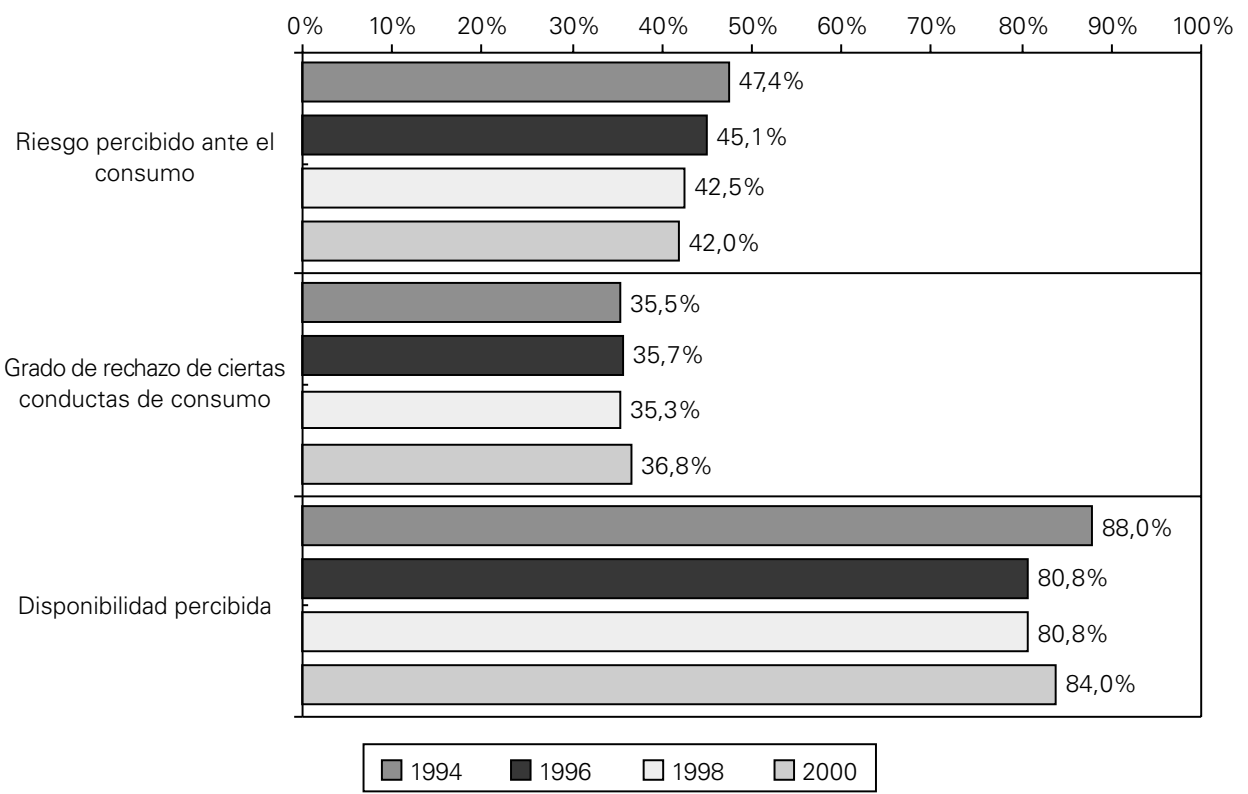

Fuente: Encuestas sobre Drogas a Población Escolar, 1994, 1996, 1998 y 2000. DGPNSD. Observatorio Español sobre Drogas.

Podrían sintetizarse las tendencias observadas en los consumos juveniles de bebidas alcohólicas en el período 1994-2000 señalando que su ciclo alcista habría tocado techo, observándose una reducción sostenida en los mismos. Este fenómeno es más positivo si se tiene en cuenta que se ha producido en un contexto en el cual los consumos de algunas otras sustancias con una importante penetración entre este colectivo (como por ej. el cannabis) han registrado una tendencia expansiva. Todo ello no debe hacernos olvidar las importantes prevalencias de consumo de alcohol que se registran en estos momentos entre los escolares españoles, así como la intensidad de los problemas asociados al consumo de bebidas alcohólicas.

\section{EL CONSUMO JUVENIL DE ALCOHOL EN EL CONTEXTO EUROPEO}

En una sociedad cada vez más globalizada y en un espacio como el Europeo, en el que 
cada vez son más evidentes los signos de integración económica, social y cultural, fenómenos como el consumo de drogas entre los sectores juveniles tienen muchos elementos comunes entre los diferentes países. Como ponen de relieve los informes realizados por el Observatorio Europeo de las Drogas y las Toxicomanías ${ }^{8}$, los consumos recreativos de drogas son una constante en los países de la Unión Europea.

Aunque la información disponible sobre el consumo de alcohol entre los jóvenes europeos es más limitada, contamos con datos pro- cedentes de estudios realizados entre jóvenes de diferentes ciudades europeas que participan de la vida nocturna ${ }^{9}$ que confirman que las bebidas alcohólicas son una sustancia habitual en la vida cotidiana y en la cultura de la diversión de los jóvenes europeos. Las diferencias entre las prevalencia de uso en España y el resto de las ciudades europeas son mínimas (Tabla 10), con una proporción de abstemios y de bebedores diarios ligeramente superior en Europa. Estas coincidencias se trasladan a las edades de inicio al consumo, que en ambos casos se sitúan en los 14'6 años.

Tabla 10. Frecuencia de uso de bebidas alcohólicas de los jóvenes que participan de la vida nocturna en Europa y España (porcentaje). 1998.

\begin{tabular}{lcc}
\hline FRECUENCIAS DE USO & EUROPA & ESPAÑA \\
\hline Nunca han bebido & $3^{\prime} 6$ & $1^{\prime} 2$ \\
Lo he probado, pero no más & $4^{\prime} 9$ & $3^{\prime} 4$ \\
Menos de 12 veces al año & $4^{\prime} 7$ & $1^{\prime} 9$ \\
Una vez al mes & $5^{\prime} 2$ & $2^{\prime} 8$ \\
Varias veces al mes & $11^{\prime} 3$ & $9^{\prime} 4$ \\
Una vez a la semana & $19^{\prime} 2$ & $27^{\prime} 5$ \\
Varias veces a la semana & $42^{\prime} 8$ & $47^{\prime} 3$ \\
Diariamente & $8^{\prime} 3$ & $6^{\prime} 5$ \\
\hline
\end{tabular}

FUENTE: IREFREA ("Night life in Europe and recreative drug use. SONAR 98" y "Salir de marcha y consumo de drogas").

\section{REFERENCIAS BIBLIOGRÁFICAS}

(1) SANCHEZ PARDO, L. Situación actual y evolución de los consumos de drogas ilícitas en España. Trastornos Adictivos, 2001, vol. 3, $n^{\circ}$ 2, pp 85-94.

(2) SANCHEZ PARDO, L. Tiempo de ocio. Guía para mediadores juveniles. Madrid: Fundación de Ayuda Contra la Drogadicción, 1998.

(3) DELEGACIÓN DEL GOBIERNO PARA EL PLAN NACIONAL SOBRE DROGAS. Encuesta sobre drogas a población escolar, 2000. Madrid: Delegación del Gobierno para el Plan Nacional sobre Drogas, 2001.

(4) ELZO, J. Sociología y epidemiología de los consumos. En: Drogas: nuevos patrones y tendencias de consumo. Madrid: Ediciones Doce Calles, 2000, pp 14-38.
(5) CALAFAT, et al. Salir de marcha y consumo de drogas. Madrid: delegación del Gobierno para el Plan Nacional sobre Drogas, 2000.

(6) ELZO, J. et al. Las culturas de las drogas en los jóvenes. Ritos y Fiestas. Vitoria: Gobierno Vasco, 2000.

(7) DELEGACIÓN DEL GOBIERNO PARA EL PLAN NACIONAL SOBRE DROGAS. Encuesta sobre drogas a población escolar, 1994. Madrid: Delegación del Gobierno para el Plan Nacional sobre Drogas, 1995.

(8) OBSERVATORIO EUROPEO DE LA DROGAY LAS TOXICOMANÍAS. Informe Anual sobre la problemática de la drogodependencia en la Unión Europea, 2000. Luxemburgo: Observatorio Europeo de la droga y las Toxicomanías, 2000.

(9) CALAFAT et al. Night life in Europe and recreative drug use. SONAR 98. Palma de Mallorca: IREFREA, 1999. 